\title{
Numerical modelling of dispersion of ammonia and chlorine in urban areas during emergency accident
}

\author{
Zdzisław Salamonowicz ${ }^{1, *}$, and Radosław Makowski ${ }^{1}$ \\ ${ }^{1}$ Faculty of Fire Safety Engineering, Main School of Fire Service, Słowackiego 52/54 St., \\ 01-629 Warsaw, Poland
}

\begin{abstract}
Modelling dispersion and determining the range of a danger zone is usually based on empirical models whose limitations simplify the result. Spreading the cloud in industrial space, urban or other irregular area with numerous obstacles, is impossible to predict using these models. The solution in this situation is the use of numerical modelling. CFD allows for consideration of complex geometry, wind flow between elements, gas weight, turbulence in the atmosphere and mass and heat flow in the test area. Such relations illustrate the phenomenon of dispersion in $3 \mathrm{~d}$ space in a very accessible way, at the same time they allow to show more precisely the characteristic places of residual gas or fast dispersion. The paper presents the possibilities of using numerical simulations for $3 \mathrm{~d}$ modelling dispersion of ammonia and chlorine in the densely built urban space of Warsaw.
\end{abstract}

\section{Introduction}

Emissions of dangerous gases and vapours into the atmosphere pose a serious threat to people and the environment. The chemical industry generates a real threat of occurrence of emergency events, the effects of which will be felt in a large area. One of the element of preparing rescue services for emergency situations is to simulate the spread of dangerous substance in the air, most often based on empirical models. Known models as gaussian, heavy gas etc. $[1,2]$ are used in risk analysis procedures. Easy and fast estimation have some major limitation: they simulate emergency scenario in open, flat terrain without any obstacles and with steady wind [3].

Another way, which shows more detailed data without empirical models limitations, is the use of numerical simulations tools. Computational fluid dynamics allows the simulation with $3 \mathrm{~d}$ geometry and with chemical and physical processes in the air [4]. The problem of numerical simulation of gas dispersion in the air was presented in many papers such as [5-8]. The numerical solution allows to visualize the dispersion of gases in the field with a large number of obstacles, taking into account the principle of energy conservation, heat and mass exchange equations or dedicated models of turbulence in the air.

\footnotetext{
*Corresponding author: zsalamonowicz@s,ssp.edu.pl
} 


\section{Research part}

\subsection{Geometry}

As the area on which numerical simulation of ammonia dispersion and chlorine modelling was carried out during emergency release, a section of the centre of Warsaw was chosen, enclosed by streets: Solidarności avenue, Jerozolimskie avenue, Marszałkowska street, Towarowa street (Fig. 1a). This area was chosen because in terms of the quantity and quality of 3-D objects in the Google Earth application, it is one of the best reflections of reality.

The creation of a three-dimensional mock-up model was a multi-stage process. Due to the complexity of the area selected for the simulation, it was impossible to create the entire geometry with ANSYS Design Modeler. The creation of the mock-up model began with the $3 D$ Ripper DX application importing the selected area from Google Earth to the Autodesk $3 d s$ Max (extensive program for creating and processing three-dimensional graphics and animations). After importing selected objects, they were repeatedly simplified to make it processable in ANSYS Design Modeler [9]. The next step after saving the three-dimensional mock-up in the .SAT extension (file extension supported by Autodesk 3ds Max and ANSYS Design Modeler) was to import the improved mock-up model into ANSYS Design Modeler (Fig. 1b). In determining the place of leakage, the focus was on the western part of the mockup model (in the scenario it was assumed that the wind would blow from west to east), taking into account possible turbulence that could cause the substance to move "upwind". Finally, the crossroads of Grzybowska and Żelazna streets was chosen as the place of the leak, as shown in Figure 1.

a)

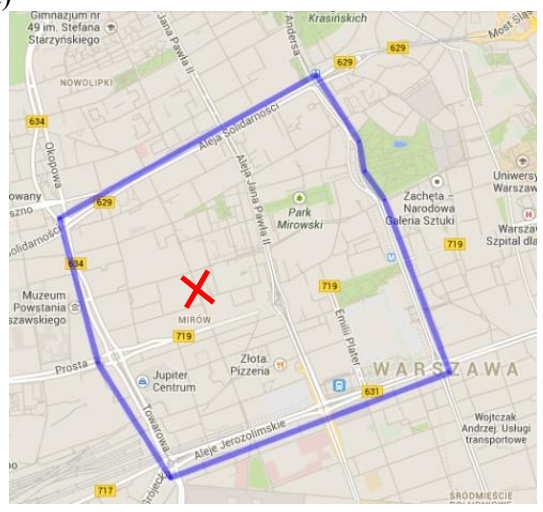

b)

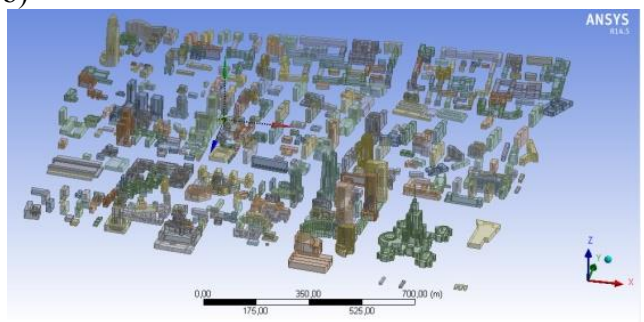

Fig. 1. Area selected for simulation: a) section enclosed by streets, b) view of the mock-up model after importing into ANSYS Design Modeler.

a)

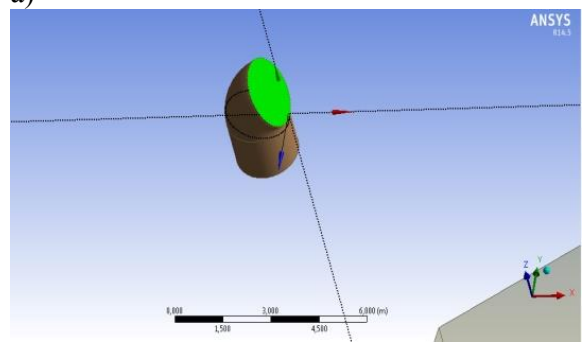

b)

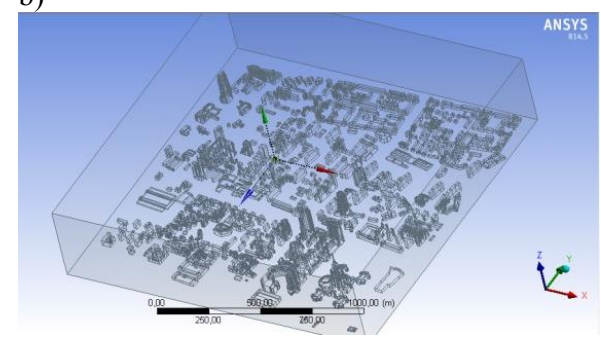

Fig. 2. The leak: a) pipe imitating the place of the leak, b) work space with the leak. 
Using the tools available in the ANSYS Design Modeler program, a pipe was created from which a hazardous substance was emitted during the simulation (Fig. 2a). This pipe was supposed to imitate the source of the leak that could in fact be a unsealed cistern, a tank or a damaged installation. The outflow area is a circle with a diameter of 150 centimetres. This surface may seem too large to imitate the above situation, but to focus on obtaining accurate results of coverage and concentration over a large area, it was decided to omit changes and behaviour of gas in the initial phase of the emission - high-flow jet outflow. Modelling of the stream outflow could be a separate issue to solve, due to the high velocity achieved by the molecules and the significance of gas density changing during outflow.

The next step in the ANSYS Design Modeler was to prepare the surface for further work. It consisted in creating a cuboid in which the entire mock-up model was placed, and then obtained a three-dimensional space in which the simulation was carried out. This area was the entire volume of the above cuboid without buildings (Fig. 2b). This was caused by the acceptance of conditions that the roughness of buildings has a negligible and irrelevant impact on the results. These buildings served as field obstacles. During the research, the air movements in the entire volume of the object were disturbed by particular obstacles, such as buildings.

\subsection{Meshing}

During the meshing process, a four-wall grid (Tetra with elements of a pile mesh) was chosen. Figure 3a shows the layout of individual grid cells. After zooming it is visible that near the objects such as the outflow pipe (Figure $3 b$ ), the cells of the grid are much smaller than the cells in the empty air spaces. Thanks to such mesh parameters, it is possible to obtain more accurate results in places (outlet pipe, building walls, terrain unevenness), where the calculations are more complicated due to obstacles and the number of variables. In addition, the size of the grid cells affects the time of obtaining results.

a)

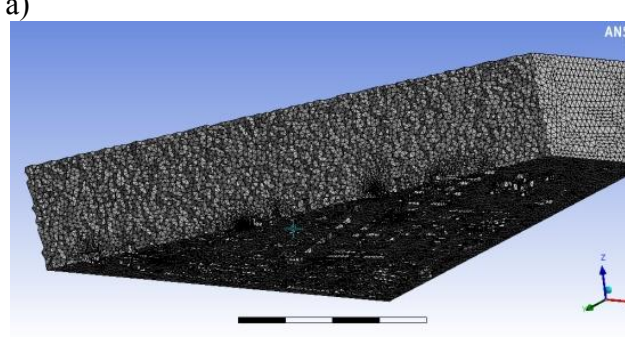

b)

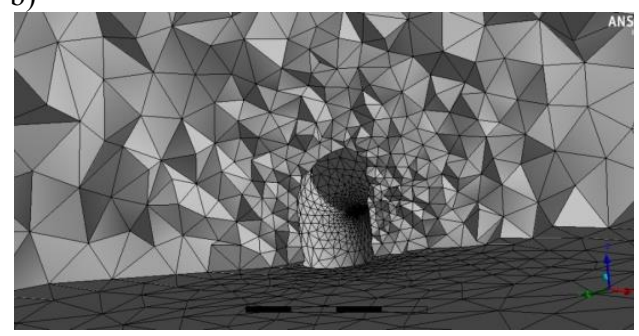

Fig. 3. View of the work space: a) from the bottom, b) near the leak.

The maximum value of the "Skewness" parameter must be less than 0.95 and the average size should not be greater than 0.33 [10]. After several cleanings and correcting of the entire grid, satisfactory results of the number of cells and the accuracy of the mesh were obtained. The cells of the grid on which the simulation was carried out mostly met the criteria mentioned above. The basic features of the grid are shown in Table 1.

Table 1. Parameters of the grid.

\begin{tabular}{|c|c|}
\hline Number of nodes & 7137680 \\
\hline The number of cells in the grid & 38579824 \\
\hline The minimum cell size & $1.589 \cdot 10^{-5} \mathrm{~m}$ \\
\hline The maximum cell size & $0.999 \mathrm{~m}$ \\
\hline Average cell size & $0.229 \mathrm{~m}$ \\
\hline
\end{tabular}


When analysing the mesh metrics, it was noticeable that a significant part of the cells in the grid are in the values described as "excellent" and "very good". Only a small amount (about 4.500 of over 2 million) of cells was considered bad results, that is, those whose size values exceed 0.95 .

\subsection{ANSYS Fluent}

For the purposes of the simulation, it was found that the surface "inlet" (the area from which the wind blows) will be the left wall of a cuboid which actually indicates the western direction. In Figure 4, this wall is shown in blue. The "outlet" area is marked by the red colour (the area that ends the field of work). Three surfaces marked in yellow, i.e. the front, top and rear faces are "symmetry" type surfaces, which means that all reactions occurring outside them are invisible and are considered symmetrical to the reactions occurring inside the cuboid. The lower plane has been described as a "wall" surface, i.e. a fixed surface below which no reactions occur.

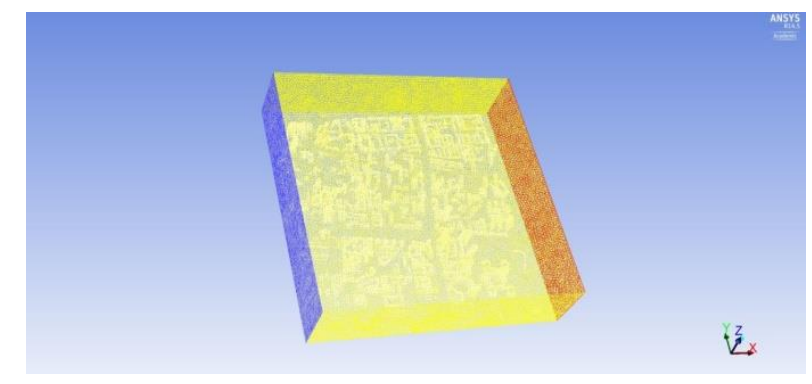

Fig. 4. View of the work field in the ANSYS Fluent program.

Three models were included in the simulation:

- Energy model;

- Transport model, which concerned only particle transport and was related to wind speed and diffusion;

- The viscosity model, in which the RNG k- $\varepsilon$ model was chosen with standard behaviour near the fixed elements (walls) [11].

Boundary condition for the air inlet was $1 \mathrm{~m} / \mathrm{s}$ and for the gas inlet $1 \mathrm{~kg} / \mathrm{s}$ (zone type was defined as "velocity-inlet"). The "outlet" zone settings determined its type as a "pressureoutlet" with pressure indicator as $0 \mathrm{~Pa}$.

\section{Results}

Presentation of the results was carried out by creating transverse or longitudinal planes depicting cross-sections of the clouds of a dangerous substance. The visualization is shown for concentrations 0 (dark blue)-500 (red) ppm. The choice of concentration range was aimed at presenting the mechanism of spreading dangerous substances and does not take into account toxic effects.

Longitudinal sections of chlorine and ammonia clouds are shown in Figures 5 and 6 . As predicted and known about the behaviour of the above-mentioned gases, chlorine as gas heavier than air almost immediately after the outflow into the workspace fell to the ground and "spilled" on the surface, the formed cloud did not exceed 15 meters above the ground. 


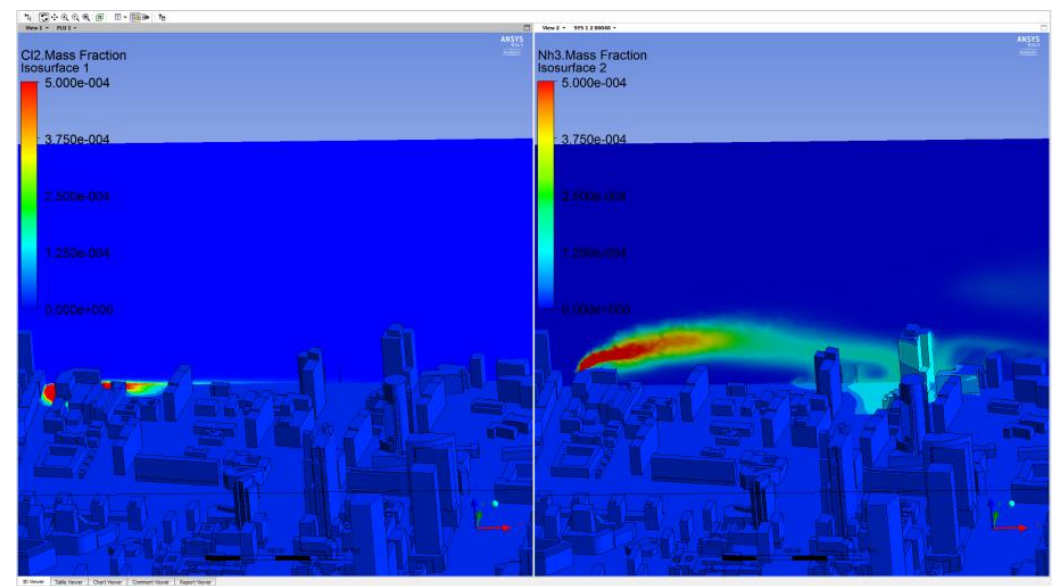

Fig. 5. Longitudinal section of chlorine and ammonia clouds.

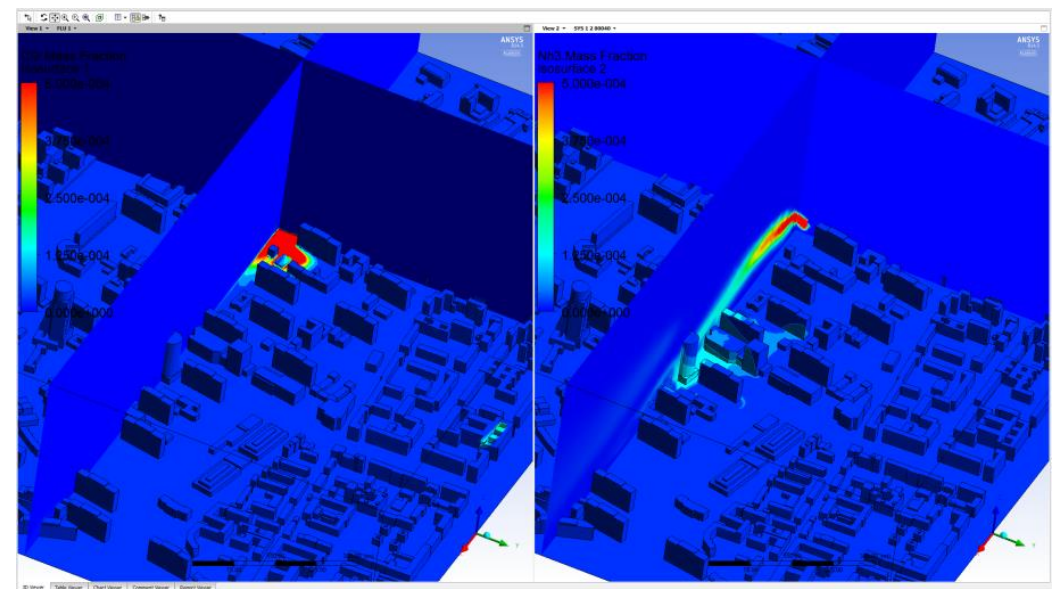

Fig. 6. A view of chlorine and ammonia clouds.

Ammonia, as a gas lighter than air, quickly rose to a height above 50 meters, where in the space above the buildings it moved along the direction of the wind. Chlorine, despite the wind blowing along the OX axis, significantly spreads to the sides (OY axis) and behaves similarly to spilled thick liquid. Ammonia rises to the top. As the concentration in the cloud decreases, it behaves like a neutral gas and spreads along the direction of the wind. The presented drawings illustrate well the impact of buildings on the dispersion of hazardous substances. In the case of ammonia, the turbulence generated by the buildings, the gas dispersion and the reduction of its concentration are very visible. In the case of chlorine, it can be observed that it spreads along the earth's surface, covering the entire space between the buildings. The presented simulation results show how the hazardous substance is spreading depending on its density.

Looking at the following projections (Fig. 7-9), the dependence of the dangerous zone size and the concentrations occurring in it from the measurement height is noticeable. In Figure 7, it is visible that in the case of chlorine, the highest concentrations occur at ground level near the emission source. The dangerous zone itself is relatively small but wide, both in the direction of wind and transverse movement. This projection confirms again the fact that chlorine is the most dangerous near the emission source, at low altitudes and at the 
ground itself. Ammonia rises to the top near emission source and increased concentrations at ground level are not observable. Due to the presence of high-rise buildings causing strong turbulence in the atmosphere, only after a few hundred meters a cloud with a concentration below $100 \mathrm{ppm}$ is noticeable. The area of the danger zone for ammonia is quite large and very irregular, but located high above the ground level.

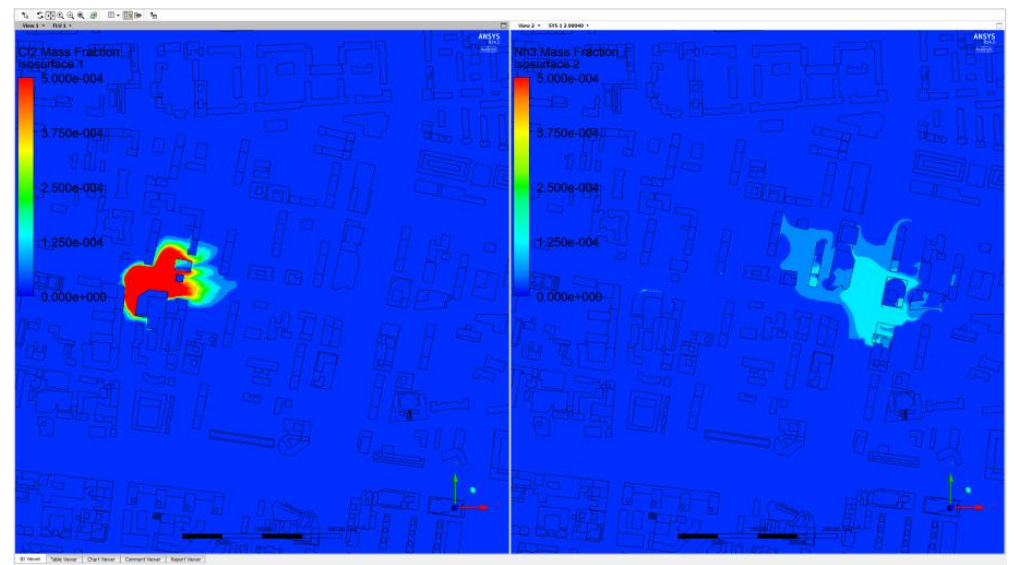

Fig. 7. Concentration of chlorine and ammonia at ground level.

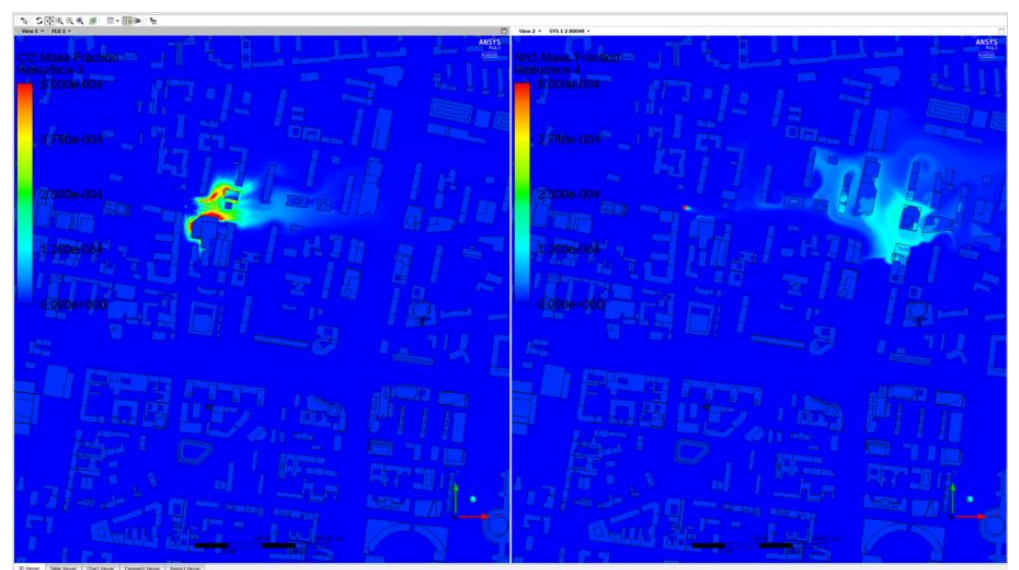

Fig. 8. Concentration of chlorine and ammonia at a height of 5 meters.

Analysing the chlorine concentration results at a height of 5 meters (Figure 8), we see a big difference compared to the previous figure. The danger zone at this height is similar in size to the zone in Figure 7, but the concentrations are much lower. It can be noticed that at this altitude chlorine mixes with air, which reduces its concentration and density. At a height of 5 meters, concentration values of $100 \mathrm{ppm}$ are achieved even several hundred meters from the emission source, which is a result of decreasing density and transport of chlorine almost as a neutral gas with a density close to air density.

The concentrations of ammonia at a height of 5 meters and the size of the danger zone is very similar to those at ground level. In addition, you can see a small field with high concentrations near the site of the outflow of ammonia. This field clearly indicates the rapid rise of ammonia as a gas lighter than air. Also greater horizontal turbulence are visible near buildings. 
At a height of 50 meters (Figure 9), chlorine is no longer present in any concentration range. However, in the case of ammonia, it is transported in a jet way, and as the distance from the source of the leak increases, its concentration decreases. Analysing the ammonia stream and its concentration at this height, one can conclude that the mixture of air and ammonia rises much higher, and from a certain height and after reducing the concentration, ammonia transport is performed as a neutral gas with a density close to the air.

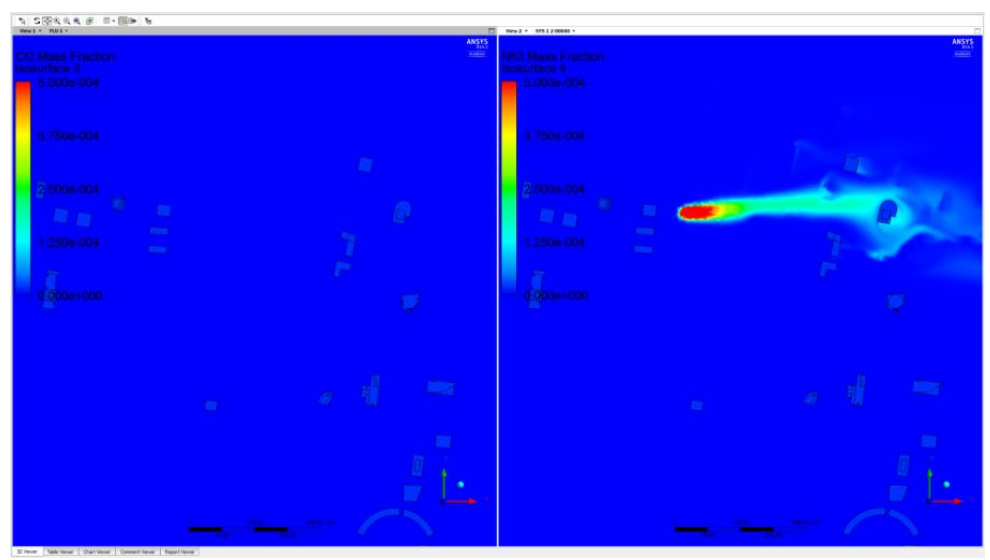

Fig. 9. Concentration of chlorine and ammonia at a height of 50 meters.

\section{Summary}

The ability of the ANSYS software to model the numerical dispersion of hazardous substances is unquestionably considered useful in the simulation of countless types of emergency scenarios. The amount of data and research methods used in the above application, gives a very large opportunity to describe variables (in time and space) of dispersion parameters of dangerous substances in the air. At the same time, the analysis of the above data and research methods gives considerable development opportunities towards the determination of the actual emission field of hazardous substances, especially in cases topographically complicated with a large amount of infrastructure. It should be noted that the magnitude of possible parameters and input data to the model can facilitate the prediction of effects in many emergency scenarios. The paper presents the idea of numerical modeling and characteristic of the research method based on the ANSYS software on the equipment of the Chemical and Ecological Rescue Division. The set goal was achieved - the possibilities of numerical modeling for simulating the emission of dangerous substance and spreading in the atmosphere were shown. This method can be applied to real areas of different size, whether they are highly urbanized areas, rural areas or small rooms equipped with installations with hazardous substances. The advantage of the developed model is that it allows you to freely shape in time and space such parameters as: the place of outflow of the test substance, wind speed, ambient temperature, type of test substance, the rate of its outflow and many others. Few difficulties were faced when writing this paper. Mainly these difficulties were caused by the research part. At the very beginning finding a source of a geometric model from which one could import a three-dimensional fragment of a densely built-up city took a long time. When the designated area was finally imported into the Autodesk $3 d s$ Max program, the structure of 3-D objects was repeatedly improved over the next few weeks because they were too complex and incorrectly constructed for the ANSYS Design Modeler program. While obtaining the results, the errors in the structure of objects made it impossible to obtain 
satisfactory results. In addition, due to the size of the work area, which in the simulation was a representation of a part of the Warsaw center in 1:1 scale (area of about 300 ha), a very powerful computer was necessary to conduct calculations. An "HP Z800" workstation was used for simulation with very good parameters on the equipment of the Chemical and Ecological Rescue Division at Main School of Fire Service, but it took several days to obtain a single result. Many times, despite many corrections, the results were not satisfactory, which required model parameters and subsequent calculations to be improved. Creating a model with such a level of complexity properly functioning in the ANSYS software requires a lot of work and a wide range of knowledge in both physics and chemistry as well as 3D object modeling and software knowledge. However, the obtained results are of very high quality, they show the occurrence of many physical phenomena and also reflect the actual topographical conditions. Due to its ability, this method is very forward-looking and should be developed. The presented examples of simulations clearly show the possibilities of using numerical solutions in the presented scenarios. Geometrically increasing computing power of computers will certainly allow faster and even more accurate modeling of such complex emergency scenarios.

\section{Conclusions}

Numerical modelling of gas dispersion in the atmosphere during emergency releases is possible, among others using the ANSYS software. The obtained results, presented graphically, in a transparent and easy way show the spreading of substances in the air. The use of two substances such as ammonia and chlorine of different densities gave very good results in the form of clearly visible differences in transport. Graphic form - cross-sections of clouds - with a concentration gradient, illustrates not only the movement of clouds but also the change in concentrations in its area. A change in the concentration of a dangerous substance in the cloud is of great importance when carrying out rescue operations.

\section{References}

1. D.B. Turner, Workbook of atmospheric dispersion estimates (EHS Ohio, 1970)

2. C.J.H. van den Bosch, R.A.P.M. Weterings, Methods for the calculation of physical effect (TNO, 2005)

3. A. Leelossy, F. Molnár, F. Izsák, A. Havasi, I. Lagzi, R. Mészáros, Cent. Eur. J. Geosci. 6, 3, 257-278 (2014)

4. A. Zieminska-Stolarska, A. Polanczyk, I. Zbicinski, J. Hydrol. Hydromechs. 63, 4, 34-341 (2015)

5. S.R. Hanna, G.A. Briggs, R.P. Hosker, Handbook on atmospheric diffusion (U. S. Dept. of Energy, 1982)

6. S.E. Kim, F. Boysan, J. Wind Eng. Ind. Aerod. 81, 145-158 (1999)

7. S.D. Sabatino, R. Buccolieri, B. Pulvirenti, R.E. Britter, Environ. Model Assess. 13, 369-381(2008)

8. Z. Salamonowicz, M. Kotowski, M. Półka, W. Barnat, B. Pol. Acad. Sci-tech., vol. 63, 1, 289-293 (2015)

9. Code ANSYS Design Modeler, www.ansys.com

10. Code ANSYS Meshing, www.ansys.com

11. Code ANSYS FLUENT, www.ansys.com 